\title{
LA PRENSA: ESPACIO DEL PODER Y FORMACIÓN DE OPINIÓN PÚBLICA EN CARTAGENA, 1920-1940
}

\author{
NEWSPAPERS: SPACE OF POWER AND CONFORMATION OF PUBLIC \\ OPINIÓN IN CARTAGENA, 1920-1940
}

${ }^{*}$ LUIS EDUARDO MESTRA NARVÁEZ

Historiador -Universidad de Cartagena, locutor de la emisora UdeC radio, de la Universidad de Cartagena. E-mail: luismestra@udcradio.com

Artículo de reflexión según clasificación Colciencias.

\section{Por: Luis Eduardo Mestra Narváez}

\author{
Artículo Recibido: Noviembre 17 de 2011. Artículo Aprobado: Febrero 05 de 2012
}

\section{RESUMEN}

La década del 20 al 40 se constituyó en un periodo de amplio desarrollo para las principales ciudades del país. Coyuntura que permitió en Cartagena el fortalecimiento de aspectos como la industrialización, la educación y el acceso masivo a la información. No obstante, dicho periodo se marcó por la legitimación de modelos de desarrollo y proyección de tribunas informativas pero bajo un tinte tradicional de camaradería política partidista. En este contexto la formación de la opinion pública en la ciudad se vio condicionada por el bajo nivel de escolaridad de la mayoría de la población y el permanente enfrentamiento de los partidos hegemónicos en el país, manifiesto en los principales perídicos de la época los cuales hacían parte de toda una infraestructura social donde la intervención de la clase burguesa local y sus ideologías políticas buscaban en lo posible atraer la balanza a su favor.

Palabras clave: Opinión pública, periódicos, ideologías políticas

\section{ABSTRAC}

The decade between the twenties and the forties became a wide developing period for the most important cities of Colombia. This situation permitted the strengthening of aspects such as industrialization, education and mass access to information. However, this period was marked by the legitimacy of development and projection models of informative platforms with a traditional hint of political party fellowship. In this context, the low level of education of most people conditioned the formation of public opinion in the city of Cartagena. The constant confrontation between the two traditional political parties, conservadores and liberales, present in the majority of the newspapers of that time, showed that their purpose was to call attention to the public opinion, in favor of one group and against the other. The political confrontation was merely a social infrastructure where the intervention of the local bourgeois, with their political ideologies, tried to gain the support for their proposals.

Keys words: Public opinion, newspapers, political ideologies

El siguiente trabajo es un primer acercamiento a lo que bien puede llamarse la historia del periodismo en Colombia y más exactamente a la forma como este se desarrolló en la ciudad de Cartagena. Para ello es necesario analizar sus antecedentes y las circunstancias en las que se desarrolló dicho fenómeno descubriendo así, el impacto ocasionado en una sociedad profundamente 


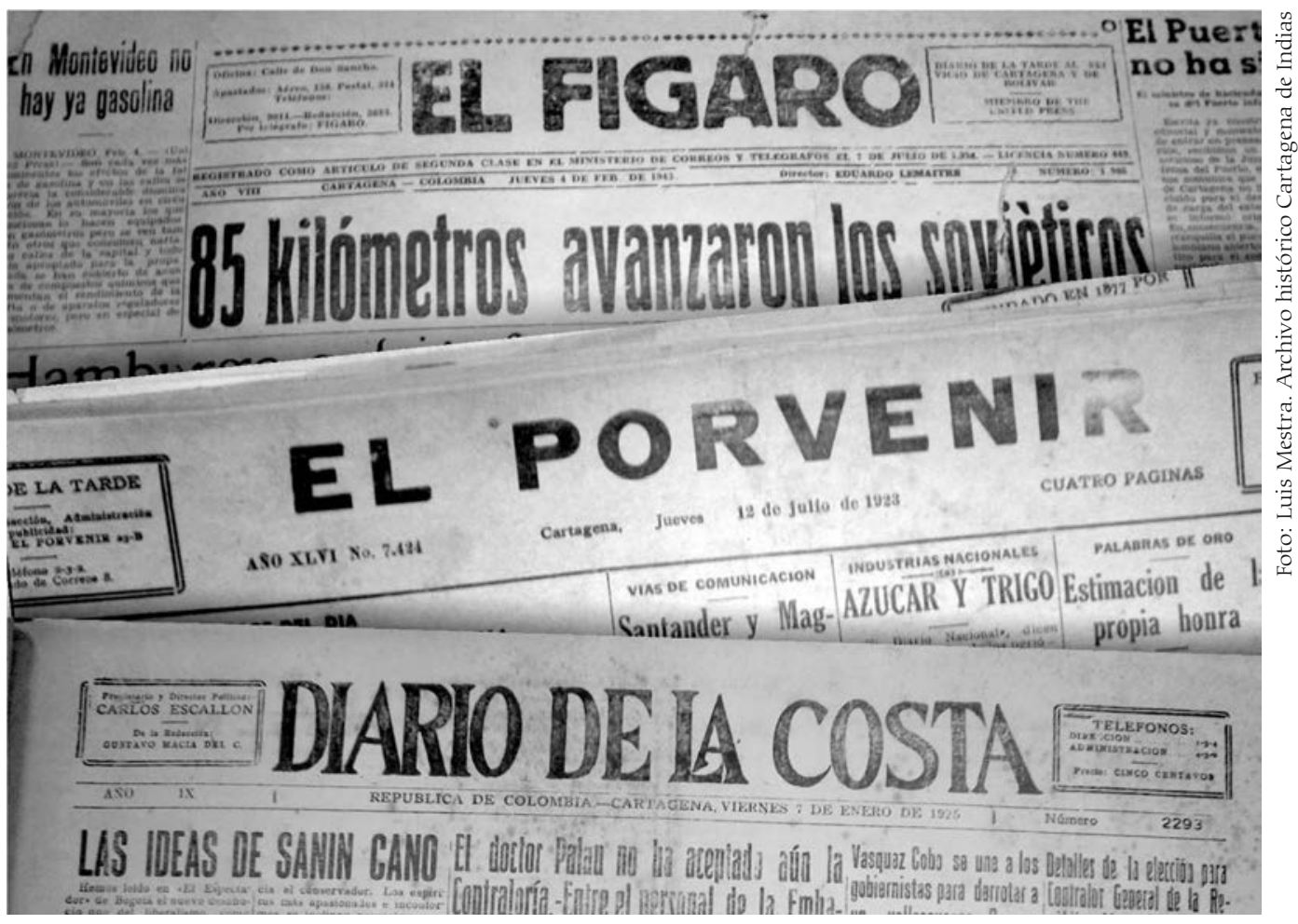

Medios de comunicación impresos en la ciudad de Cartagena

aferrada a la tradición oral y a la vida rural. En medio de este contexto, resulta interesante observar las condiciones que rodearon a la creación de un espacio de opinión pública en la ciudad y la forma cómo la élite local halló cabida para expresar sus intereses de clase y el discurso que legitimaría su intervención en las transformaciones que experimentó Cartagena entre las décadas del 20 al 40. La opinión pública (lat. Opinio publicus, conjeturar, dar a parecer oficialmente) se entiende -de acuerdo a lo que afirma Fraçois Xavier Guerra- como "la expresión pública de las ideas que permite la libre discusión sobre toda temática, incluida la política. (Ella) existe cuando... una instancia moral independiente del Estado... juzga en nombre de la razón, validez no solo de las medidas del gobierno sino también de los principios generales que deben regir la sociedad"1.

La prensa como factor que construye opinión, se debe entender como una maquinaria que "incesantemente recibe y devuelve impulsos, consume y reproduce ideas y esfuerzos; refleja y es reflejada, condensa y vulgariza obedeciendo a fuerzas centrípetas y centrífugas". Incluye lo nacional, lo extranjero, lo político y lo social habiendo sido según Gustavo Otero "la mayor tribuna de doctrina democrática y nuestro mayor estímulo de producción literaria. Es sin duda Historia del día y fuente de variadas noticias para la posteridad" 2 . Por todos estos

Santos, Adriana. "La prensa católica en el estado soberano del Magdalena: guerra de palabras y pedagogía política" en: el taller de la Historia, no 2 , Universidad de Cartagena, Facultad de Ciencias Humanas, 2002, pág. 86.

2 Otero Muñoz, Gustavo. Historia del periodismo en Colombia, Selección Samper Ortega, Santa Fe de Bogotá, 1948, p. 171 
aspectos resulta imprescindible rastrear a quienes escribían en los periódicos y para quienes lo hacían. Cómo reflejaba la realidad y en qué circunstancias la presentaban al público y por último, determinar sus implicaciones en el imaginario de sus lectores y en los hechos importantes de la historia nacional.

Si se enmarca este ensayo dentro de la historia de las mentalidades y la larga duración es fundamental la identificación de cuales son y han sido los agentes y/o factores que permiten la construcción y el fortalecimiento de imaginarios en una sociedad determinada, donde el comportamiento y la materialización de los pensamientos de un individuo están expuestos a una serie de normas prefijadas que buscan la unificación de criterios a través de instrumentos establecidos por el Estado. El sistema educativo y demás estamentos civiles y religiosos incluso la prensa, se conviertan en componentes cruciales para el logro de este objetivo. Lo curioso es que toda la infraestructura del Estado fácilmente es permeable a la intervención de una clase social alta que buscará en lo posible atraer la balanza a su favor. En Europa, dicha clase social nació y se fortaleció con la Ilustración y se cimentó con el Capitalismo y para el s. XX ya había alcanzado la mayoría de edad, la autonomía y el espíritu de liberación necesarios para dirigir el Estado en lugar de la nobleza. ${ }^{3}$

La burguesía propuso el nacionalismo como elemento crucial para la modernización de las estructuras gubernamentales y también inició una campaña de "reculturización" de las clases populares, formalizando así, la educación obligatoria, y a su vez, un sistema comunicativo a través de la introducción a la lectura y la consecuente creación de un mercado para la prensa de masas ${ }^{4}$. Todo este marco permitirá comprender en que contexto surge el nuevo concepto del periodismo que se manejará durante el S. XIX y la primera mitad del S. XX y por lo tanto, la función que los medios impresos y más tarde los audiovisuales cumplirán. Los periódicos como mecanismos de difusión de ideas ya habían nacido siglos antes pero solo durante ese período ampliarán sus rango de acción, secularizarían su función y servirían de canal para la transmisión de intereses claros por parte de las élites nacionales.

${ }^{3}$ "Martínez, Germán y otros sofía de la Ilustración en: la filosofía en Colombia historia de las ideas, El Búho, edit., Bogotá, 1992 pág. 139.

${ }^{4}$ Álvarez, Jesús. Ascención Martínez. Historia de la prensa hispanoamericana colecciones MAPFRE. Edit. Madrid, 1992, pág. 11.
La burguesía convirtió a la prensa en la herramienta por excelencia al servicio de poder capaz de conmover y en lo posible, transformar las estructuras del pensamiento de una época determinada. Una de sus obras cumbres se registró a partir del siglo XVIII con la conformación de los Estado-Nación modernos producto de toda la imaginería y la acción desplegada por una clase burguesa ilustrada, que para el caso de Europa, se esforzó por aprovechar 
la coyuntura cultural y social imperante en el momento para difundir a la población en general un proyecto de nación que acabaría, en teoría, con un régimen absolutista que coartaba el nacimiento del proyecto nacionalista como reacción al absolutismo integrando no solo principios económicos sino también, principios ideológicos y emocionales. ${ }^{5}$ De esta manera, a lo largo de los países del antiguo continente se difundieron frases como: "Hemos hecho Italia, ahora tenemos que hacer italianos", expresiones que en sí, reflejaban un objetivo claro para lo cual, sería necesario utilizar herramientas que permitieran según Hobsbawm, crear una "propaganda de nacionalismo" que atrajera la atención de toda la población.

Hasta mediados del siglo XIX la difusión de estas ideas se hizo a través de los diarios nacionales y otros tipos de literatura donde el discurso político se manifestaba de manera implícita pero de igual forma, las escuelas, los centros culturales, los cargos públicos y el ejército, se consolidaron también en el complemento eficaz para llevar a cabo una empresa que pretendía la creación de un "idioma nacional" entre las masas, "porque las escuelas y demás instituciones al imponer un idioma de instrucción, imponen también una cultura, una nacionalidad"6. Es así como el sistema educativo y los medios de comunicación se constituyen en herramientas de poder al servicio del poder. No obstante, esta visión implicaría asumir una interpretación de carácter unidireccional donde el pueblo como agente social solo cumpliría un papel pasivo.

Peter Burke al referirse a la introducción de la imprenta en las sociedades del Antiguo Régimen, afirma que la cultura oral y la organización de las clases populares eran, y se puede decir que aun son, lo suficientemente fuertes como para resistir la simple corrección y estandarización desde arriba ${ }^{7}$. Pero lo que si resulta evidente es que la prensa y más específicamente la creación de la imprenta, ofreció a las sociedades del mundo una serie de códigos prefijados que a su vez, le permitieron al pueblo tener una visión más general de la realidad que le rodeaba. Por otro lado, frente a la posible pasividad de los lectores, Roger Chartier agrega que existe una especie de negociación entre estos y los autores de los textos, por lo cual el pueblo viene ahora a dotarse de una actitud más activa frente a lo que lee o escucha ${ }^{8}$.

Con el proyecto liberal del s. XIX en la gran mayoría de los países occidentales se implementó un programa que democratizara la educación e introdujera la lectura en medio de las clases populares mediante la difusión de estampas, libros de cordel, periódicos populares junto a las novelas, revistas... obras

5 ibid. P 10

${ }^{6}$ Hobsbawn, Eric. "La construcción de las naciones" en: La era del capital, 1848 - 1875 . Buenos Aires, Crítica, 1998, págs. 101 y 107.

7 Burke, Peter. La cultura popular en la Europa moderna. Alianza Editorial, Madrid, pág. 224.

${ }^{8}$ Chartier, Roger. El mundo como representación. Gedisa, Madrid, p. 38-39. Ver Zenon Davis, Nataly. "La imprenta y el pueblo", en: Sociedad y cultura en la Francia Moderna, Crítica, Barcelona, . La autora explora la forma como la imprenta influyó en los sectores populares de la Francia del s. XVI, describiendo los formatos, el público y los temas que hablaban (pasquines, poemarios,...) 
de vulgarización. Se buscaba entonces, que la gran mayoría de la población tuviera acceso a los principios de igualdad ciudadana que propugnaba el Liberalismo. Cuando se habla de democratización se entiende como ese proceso en el cual cada hombre y cada mujer pueden ser más los sujetos de su destino, "singularidad y compartida... y en lo posible resistir algunos procesos de marginación a ciertos mecanismos de opresión" 9 .

En lo que respecta a Hispanoamérica, después de 1810, la prensa empezaría a convertirse en una herramienta útil en la legitimación de un sistema de gobierno constitucional. No obstante, elementos como el analfabetismo, la presión del gobierno español en América, y el enraizado conservadurismo de algunas capas sociales frente a las ideas que representaban "un peligro" para la estabilidad del Estado, no permitieron que su difusión se diera paralela al proceso llevado a cabo en el Antiguo Continente. Sin embargo, la historia ha confirmado que en Colombia y el resto de Latinoamérica la prensa escrita además de garantizar la creación de un espacio público de opinión, ha venido cumpliendo con su papel legitimador de los discursos políticos de las clases dominantes. No necesitó de la creación de un nuevo modelo para regular la información debido a que el modelo inglés definido por "El Libel Act de 1992" reconocía la libertad de expresión como principio de derecho y base de las nuevas sociedades facilitando a los gobernantes las herramientas que ayudaran a mantener un control férreo sobre la misma y limitar el exceso de temores de la oligarquía terrateniente. ${ }^{10}$

\section{LA IMPRENTA: Génesis de la información masiva}

El primer capítulo de la historia de la Imprenta en Occidente, se registra en Gutemberg, Alemania, en 1456, año en que se editó la primera Biblia en 48 líneas impresas. En España, 19 años más tarde, aparecería el primer texto con canciones a la Virgen. No obstante, con la Contrarreforma y la consecuente imposición de la Inquisición, el 8 de julio de 1502, se estableció un fuerte control sobre su uso. Todo este fenómeno ayudaría a que dicha intervención estuviera íntimamente ligada al mundo religioso durante gran parte de los siglos XVI y XVII. En América, el control de la imprenta se convirtió en una prioridad para las autoridades hasta el punto de hacerla una herramienta

${ }^{9}$ Petit, Michele. Lectores: De espacio íntimo al espacio
público. Fondo de Cultura Económica. México, 2001, pág. 104.

10 Álvarez. Op. cit, pág. 11

${ }^{11}$ Ibíd. pág. 21 al servicio de propósitos políticos, de tal manera que las leyes y demás normas contenidas en los documentos oficiales transmitieran los valores que sustentaban la monarquía universal española y de esta manera se constituyó en un instrumento más de dominación ${ }^{11}$. 
De acuerdo a la Asociación Mundial de Periodistas (WAN), el primer diario publicado en el mundo comenzó a circular en junio de 1605 en Estrasburgo, bajo el nombre de "Ralation", editado por Johann Carolus, quien lejos de servir a la Iglesia o al Estado, de forma independiente se ganaba la vida a principios del siglo XVII elaborando boletines informativos manuscritos, que vendía a muy alto precio a varios abonados pudientes. En ellos, se reproducían noticias enviadas por una red de corresponsales también pagos. En 1604 compró una prensa integral, y en el verano de 1605 empezó a imprimir sus propios boletines, debido a la demora que implicaba realizarlos a mano ${ }^{12}$.

En Hispanoamérica la introducción de la imprenta corrió en la gran mayoría de los casos por cuenta de las comunidades religiosas. En México llegaría en 1535-36, por iniciativa del Obispo Juan Zamarraga y del Virrey Antonio Mendoza. Los Jesuitas, por otro lado, se encargarían de las primeras imprentas del Perú (1580-83), el Alto Perú, el Río de la Plata (1700), en Chile, en la Nueva Granada y en Quito (1755). Los Franciscanos se encargarían de la Capitanía de Guatemala (1657-60) y El Salvador. En las Antillas, Cuba sería la primera en el Caribe en tener una imprenta por iniciativa de Carlos Haba; por otro lado, Puerto Rico debía esperar hasta 1806 para tener su primera imprenta al igual que la Capitanía de Venezuela, donde después de muchos conflictos entre la clase intelectual y el gobierno español, por fin pudieron publicar los primeros textos en 1808. Dichos textos impresos se relacionaron en principio con el culto mariano, la catequesis y las misiones hacia zonas de indios bravos. Ejemplo de ello fueron "La primera edición de la doctrina católica" en el Perú, "La escuela espiritual" en México, y el "Septenario al corazón doloroso de María Santísima"13.

Durante la Colonia, el periodismo se convirtió en un fenómeno de carácter urbano y selectivo que solo se presentaría en los principales centros administrativos de América, con el fin de dinamizar los asuntos relacionados con la Iglesia y la Corona Española. Las primeras expresiones del periodismo fueron "las hojas de volantes" o "relaciones" donde se registraban acontecimientos especiales con tendencias a la exageración.

La primera relación que circuló en América relataba los hechos ocurridos alrededor del terremoto de Guatemala entre 1541 y 1542, y más tarde aparecerían en Perú. Le siguieron los "boletines" o "noticiarios" los cuales se editaban en Madrid y registraban sucesos locales y extranjeros acompañados con algo de publicidad. Las noticias luego pasaron al papel periódico - "Los

${ }^{12}$ El Universal, No $^{9} 993$ Junio 12 de 2005 .

13 Álvarez. Op. cit. pág.

Palobra No. 12. Agosto de 2010 - Julio de 2011 
${ }^{14}$ Ibíd, pág. 34 y 39.

15 Álvarez. pág. 179.

${ }^{16}$ Ibíd, p. 54.

${ }^{17}$ Pese a que Joseph Rioja afirma que en Cartagena existió una primera imprenta para 1769 sería precisamente la de Antonio Espinosa de los Monteros la primera en ser utilizada en la ciudad durante la segunda mitad del s. XVIII Espinosa, quien tuvo su taller al sur de la Calle del Tablón hasta que el virrey Flórez decidió llevárselo para Santa Fe de Bogotá, dejó evidencia de su trabajo por lo que se considera como el pionero de esa materia en Colombia. Luego de este evento el Consulado de Comercio de Cartagena realizaría la petición para hacer uso de una nueva imprenta traída de Madrid en 1800 , pero entre los conflictos desarrollados por el virrey del momento, y la tardanza de la resolución del caso por parte del rey, solo 12 años después sería utilizada para escribir la primera constitución de Cartagena. Lemaitre, Eduardo. Historia general de Cartagena, tomo IV. Banco de la República, Bogotá 1983, p. 403-405.
Avisos" (Perú, 1621-22)- sirviendo de tribuna de expresión para una especie de criollismo durante el s. XVII. La primera "Gaceta" en América se registraría en México en 1666. Este tipo de material impreso era recibido en los puertos y dirigidos a una minoría de la población, debido a la falta de un mercado de lectores potenciales y al reducido número de imprentas que funcionaban en Hispanoamérica. Más tarde con la Ilustración, también se importaría la censura y la Inquisición ${ }^{14}$. La prensa ilustrada criticó las medidas borbónicas y propició una filosofía aristocrática de la sociedad y difundió temas sobre la literatura, la política, la filosofía, economía y la ciencia. En la Nueva Granada el primer periódico ilustrado aparecería en 1739 de la mano de Manuel del Socorro Rodríguez.

La primera vez que se habló de una especie de unificación de la opinión pública en la historia del periodismo se registró entre 1808 y 1810 como consecuencia de la invasión napoleónica a España, lo cual motivó el rechazo generalizado de toda la élite criolla y a partir de allí, solo se habla de procesos con destinos diferentes. Durante el siglo XIX, posterior a la independencia, la prensa se volvió mucho más política. Fue un instrumento personal para aquellos que deseaban alcanzar el poder y por ello, los periódicos que existieron tuvieron una vida efímera por estar a merced de los vaivenes de los acontecimientos políticos ${ }^{15}$. Sin duda, fue un medio de expresión capitalizado por sectores minoritarios que lo utilizaron como "instrumento de comunicación y a la vez de control social"16 pero regulada por juntas de censuras.

Las primeras imprentas eran demasiado rudimentarias y sus elementos tipográficos muy pobres para la impresión de libros. En 1780, el virrey Flórez hizo traer de Cádiz, una nueva tipografía que llegó en veinticuatro cajones, y en 1782, comenzaron a aparecer ediciones con el nombre de Imprenta Real, que reunía en una sola tipografía las tres prensas: la antiguamente utilizada por los Jesuitas, la llevada de Cartagena por el virrey Flórez y la nueva importada de España. En ella, bajo la dirección de Antonio Espinosa de los Monteros $^{17}$, se editó el primer libro colombiano, la Historia de Cristo Paciente, en dos tomos por José Luis Azula (1787). A raíz del terremoto de 1785, se imprimió también en la Imprenta Real un boletín sobre el suceso, que llegó a ser la primera publicación noticiosa, seguida a los pocos días por la Gaceta de Santa Fe, primer intento de periodismo que alcanzó a publicar tan solo tres números. 


\section{Los medios impresos: el caso de Colombia}

El verdadero periodismo en Colombia nació con la publicación del Papel Periódico de la ciudad de Santa Fe de Bogotá, cuyo primer número apareció el 9 de febrero de 1791. Este semanario fundado y dirigido por el literato cubano Manuel del Socorro Rodríguez (nacido en Bayamón) era de ocho páginas en formato de octavo. Se editaba en la imprenta que dirigía Espinosa y estuvo publicándose con regularidad durante más de cinco años (hasta el número 270). Se dirigía a un público caracterizado por la "prudencia y la sabiduría" entre los que se encontraban, de mayor a menor proporción, funcionarios, oficiales militares, colegiales, clérigos y comerciantes ${ }^{18}$. En los últimos días coloniales, en nuevas tipografías importadas de Estados Unidos, se editaron otras publicaciones periódicas como la Bagatela de Antonio Nariño, y el Semanario del Nuevo Reino de Granada, de Francisco José de Caldas.

De 1824 a 1850, se inició con la organización de la República y el periodismo, fue un período caracterizado por la fragmentación del poder, el caudillismo y gobiernos cortos. En 1848 y 1849 se formalizarían los partidos políticos tradicionales del país, dos formas distintas de defender los intereses de los grupos dominantes, y que en definitiva lo postulaban a través de la prensa y otros medios. Ellos intentarían fundamentarlos teóricamente pero se entrecruzarían en la mentalidad de los dirigentes republicanos. La prensa en general, participó en los logros y fracasos por construir la República. Durante las primeras décadas del s. XX, la concepción del periodismo pasó de ser simplemente político a una versión más comercial pero sin dejar de lado la afinidad partidaria aunque siempre se presentaban como independientes, siendo aún pro gubernamentales. Ahora, como empresa, el objetivo sería la rentabilidad.

En Colombia, la década del 20 empezaría con transformaciones de carácter político a nivel del gobierno del país: Marco Fidel Suárez dejaba la presidencia el 11 de noviembre de 1921 en manos de Jorge Holguín, como resultado de las presiones de sus opositores quienes vieron en la crisis fiscal del país y en las negociaciones del Tratado con Panamá, los argumentos necesarios para deponerlo de su puesto. No obstante, entre sus logros estaba el decreto 599 de marzo de 1920 que dio paso a la creación de la aviación en Colombia. Fue así como el 16 de febrero de 1920, los cartageneros vieron volar por primera vez el avión F40 y según la descripción del periódico El Porvenir en su emisión vespertina, alcanzó a dar varias vueltas alrededor del Monumento a la Bandera;

18 Silva, Renán. Prensa y revolucionaria a finales del s. XVIII. Banco de la República, Bogotá. 1988 pág. 33. 
seis días más tarde se registraría el primer vuelo comercial BarranquillaCartagena $^{19}$. El país iniciaba también, un proceso de modernización que se traducía en la construcción de vías, líneas férreas, puertos y creación de instituciones administrativas y de servicio público que daban apertura a un cambio de conciencia y estilo de vida de los colombianos. La prensa nacional no podía dejar de registrar este proceso.

\section{Cartagena de Indias y su periodo de modernización}

La hegemonía conservadora llegaba a su fin en 1930 y empezaba un gobierno liberal que se extendería hasta 1946. Durante ese período, Cartagena fue también escenario de transformaciones de carácter económico y social. Años antes había llegado la luz eléctrica y el agua (proveniente de Matute, y más adelante de Gambote), se habían embellecido los parques y plazas adornándolas con monumentos de próceres para lo cual se utilizó el bronce y el mármol traído por Juan B. Mainero. Además se dio la inauguración del mercado público y el ferrocarril. Por otro lado, hubo un mayor desarrollo del transporte, construcción de edificios y complejos urbanísticos importantes como también, la reapertura de la actividad portuaria a partir de los años 30.

De igual forma se registraba un leve incremento de la industria local. Empresas como la de los señores Daníes y Cía., la fábrica de hielo Walter, la fábrica de los señores Merlano y Cía., la mueblería y las fábricas de velas y chocolates de los señores Lequerica Hnos., entre otras ${ }^{20}$, dinamizarían la economía de Cartagena desde muy temprano en el siglo XX pero también destacarían la importancia de "ilustres familias" relacionadas con las actividades comerciales determinadas entre ellos, los Román, los Lemaitre, y los Emiliani, quienes tenían a su cargo las empresas de químicos, artesanías, cigarrillos y puntillas; los De la Espriella con la industria de la ropa interior y el calzado; los Merlano con la industria tejidos y chocolates; los Araujo Jiménez con las empresas de ladrillos, cementos y jabones, la encuadernación y la tipografía. Estas últimas, las realizaban en compañía de los Mogollón; los hermanos Benedetti con su empresa panadera, y la explotación pesquera ${ }^{21}$.

19 Lemaitre. Op. cit. págs. 406-420.

${ }^{20}$ Ibíd., pág. 483.

${ }^{21}$ Ibíd., págs. 555-556
Todas estas familias tuvieron alguna injerencia en el proceso de modernización de Cartagena razón por la cual, no se pone en duda su influencia en la prensa de la época. Ellos eran la "clase burguesa y educada" llamada a impartir el proyecto político y social que en algún momento se aplicó en Europa. Solo basta con 
mirar el listado de colaboradores que tuvieron los principales periódicos de la ciudad -en su gran mayoría conservadores- para darse cuenta en manos de quien estaba la labor de construir un "espacio de opinión pública". Ellos tenían los medios políticos y económicos suficientes para intervenir en todas las esferas de la sociedad de la época y en este sentido, la prensa se convierte en el reflejo de esa realidad, y el escenario de los choques discursivos de los dirigentes políticos en este período tal como lo refleja un estudio realizado por Adriana Santos acerca de la guerra de palabras entre la Iglesia y los partidos políticos pero para el caso del Magdalena. ${ }^{22}$

El periódico El Porvenir de Cartagena tenía entre sus páginas una sección dedicada a "la política conservadora" donde se anunciaba todo el acontecer relacionado con los miembros de ese partido y las actuaciones que cada uno de ellos realizaba en todo el departamento. Desde dicho espacio se afianzaban las relaciones sociales, se presentaban condolencias y se autoalababan los integrantes de la bancada conservadora. Frente a los comicios se autocatalogaban como "los amigos de la legalidad, los apóstoles de la pulcritud... los eternos incendiarios de respeto a la ley" ${ }^{23}$, expresión que resultaba luego de descubrirse un fraude electoral en contra de sus candidatos a la Asamblea Departamental, motivo por el cual, el periódico El Porvenir en su emisión del 15 de enero de 1925, se complacía en la lista de candidatos y recomendaba de manera especial a los conservadores y al pueblo en general-como una especie de prensa eleccionaria- votar por "aquellos gallardos paladines, conservadores auténticos, y de acción que en el salón amarillo sabrían dirigir"24. Por ejemplo, el 8 de mayo del mismo año, el director de la Junta Conservadora de Bolívar conformada en ese entonces por los señores Carlos Vélez, José María de la Vega, M. M. Torralba, entre otros destacados personajes de la vida política local, anunciaba a los cartageneros el fallecimiento de "edad muy madura" del "esclarecido ciudadano don Bartolomé Martínez Bossio" comerciante y dueño del Banco (de) Cartagena, ubicado donde actualmente funciona la biblioteca pública Bartolomé Calvo. Y sigue diciendo:

“... que tan benemérito patricio que honró con sus piadosas virtudes y altas dotes financieras a Cartagena, su ciudad natal, y al país, conquistando puestos prominentes en el comercio y la banca, ora estableciendo importantes empresas industriales que hablaban muy alto de sus renombradas capacidades y que ha contribuido al desarrollo del comercio y de nuestra riqueza nacional..."25
22 Santos, Adriana. "La prensa católica en el Estado Soberano del Magdalena: Guerra de palabras y pedagogía política. En: Taller de la Historia no 2 Iniversidad de Cartagena, Gacultad de Ciencias, Humanas, 2002, págs. 85100.

23 AHC. El Porvenir. 16 de febrero de 1925

${ }^{24}$ AHC. El Porvenir. Jueves, 15 de enero de 1925

${ }^{25}$ AHC. El Porvenir, no 7942, mayo de 1905. 


\section{La prensa cartagenera: Entre el bipartidismo político y la libertad de expresión.}

En un período de grandes transformaciones políticas y económicas, Cartagena contaba con una nutrida lista de periódicos de una clara identificación ideológica. Para el período de 1920 a 1940, circulaban en la ciudad varios medios impresos con una filiación política definida entre ellos: El Porvenir (1877-1928) fundado por Antonio Araujo León y también la principal tribuna y órgano de expresión del movimiento regeneracionista durante el mandato de Rafael Núñez. En las primeras décadas del siglo XX, es claro que su filiación tendió más hacia la ideología conservadora. El Diario de la Costa, (1921/1926 - 1980) de filiación conservadora, fue fundado por el señor Gabriel Eduardo O’Byrne y se convirtió en la escuela de periodismo más importante de las nuevas generaciones de Cartagena.

Por otro lado, El Mercurio (1927-1934) de filiación conservadora, era catalogado como el periódico mejor editado de toda Colombia en la época. El Fígaro (1926-1948) bajo la dirección de don Lázaro Espinosa se constituyó en la tribuna más importante de oposición liberal durante sus años de circulación en Cartagena. Pero existían otros no menos importantes como lo fueron: La Época (conservador), La Patria (liberal moderado), La Opinión (conservador), El Combate (liberal), entre otros. Todo lo anterior permite comprender que la prensa de la época no solo cumplió con su papel informador, sino que también se consolidó en la herramienta política por excelencia para expresar y fundamentar los intereses de partido de la élite cartagenera y también, en el mecanismo eficaz de oposición frente a la actuación del partido contrario, fenómeno que se agudizaba mucho más en vísperas de los comicios para los principales cargos del gobierno en Colombia ${ }^{26}$.

26 En Octubre de 1925, el partido Conservador anunciaba a los cartageneros la aplastante derrota que había sufrido el partido Liberal a nivel de todo el departamento, en los comicios que escogerían a los representantes al concejo de la ciudad, pero también los acusaban de tambien, los acusaban de cometer fraudes electorales tales como el "trasteo de votos" y de engañar a muchos campesinos con botellas de alcohol para que cometieran actos delictivos y promovieran el desorden en varias zonas del país, en varias zonas del país, y Barranquilla donde se registraron varios muertos Ver: AHC. Diario de la Cosa, $n^{\circ} 2507,5$ de octubre de 1925

${ }^{27}$ AHC. Diario de la Costa, $n^{\circ} 2508.6$ de octubre de 1925 .
Durante el periodos de Hegemonía Conservadora (1886-1930) y la Liberal (1930-1946) era inevitable que en la prensa local no se ejerciera una presión desde arriba sobre el partido opositor. Ejemplo a ello, El Porvenir anunciaba en mayo de 1925 "el triunfo absoluto" del partido Conservador en todo el departamento de Bolívar, y "la aplastante derrota del partido Liberal". El Diario de la Costa, en octubre del mismo año anunciaba nuevamente "el resonante triunfo conservador" en los comicios para escoger a los representantes al Concejo de Cartagena, no sin antes acusar directamente al partido político contrario como una banda de "criminales, deshonestos y manipuladores" y principales culpables de las masacres ocurridas en Calamar y en Barranquilla el mismo día de las votaciones ${ }^{27}$. 
Sí el discurso manejado en la prensa ayudó a la legitimación de los modelos de desarrollo de la ciudad y resaltar también la figura de importantísimos empresarios bajo un tinte de camaradería partidaria vale la pena reflexionar sobre las siguientes cuestiones: Cómo fue el comportamiento de la población frente a ellos, qué parte de la población

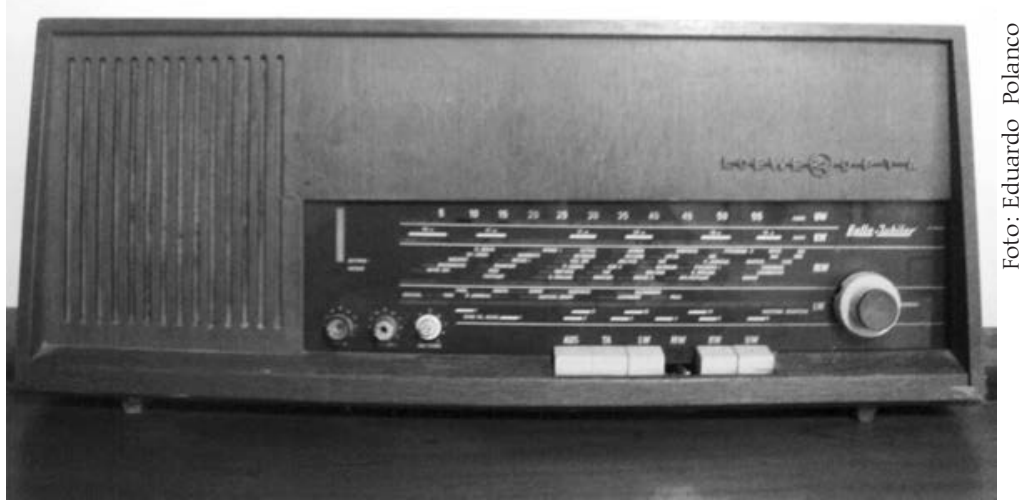

Radio Trans-Oceanico tenía acceso a esta información, cómo estaba el nivel de alfabetización para el período en cuestión y quiénes podían opinar a favor o en contra de ello.

En lo que respecta a Colombia, para el principio del siglo XX, aún seguía siendo una sociedad de carácter rural con una población campesina en su mayoría analfabeta cuya movilidad campo-ciudad resultaba siendo escasa, lo cual era reforzado también por la limitada existencia de una industria nacional fuerte, capaz de atraer a las grandes masas hacia los principales núcleos urbanos. La política liberal -a partir de 1930- contenía un programa educativo que buscaba "democratizar la enseñanza en Colombia" pero acompañada de una preparación técnica con la cual los jóvenes pudieran desempeñarse en oficios $\operatorname{varios}^{28}$.

Años antes, y pese a la crítica situación de la educación en el país, las autoridades del Departamento de Bolívar anunciaban que el balance en cuanto al número de profesores y escuelas en 1927, demostraba el ascenso de la institución pública en la región, y aunque no alcanzaba el nivel del Departamento de Caldas, catalogado como el mejor organizado en esta materia, los avances eran satisfactorios. Este informe sería una respuesta a un artículo de un periódico local que calificaba la instrucción pública de Bolívar como "lamentable" y para refutar dicha versión se mostraron en El Porvenir los registros de 1923-1926, donde se presentaba un incrementos sustancial en el número de escuelas públicas, que pasaron de 346, con 14.461 alumnos matriculados y 535 maestros, a 414 escuelas, con 22.793 alumnos y 544 profesores $^{29}$. Pero en realidad este dato no puede ser garante de la verdadera realidad de la educación en Bolívar, debido a que es necesario un mayor estudio sobre el tema. De acuerdo a lo que afirma Renán Silva, el nivel de analfabetismo se mantuvo en la segunda mitad del siglo XX por encima del 40\%, con una diferencia marcada entre la ciudad y el campo y menos marcada entre hombres

28 (N.A.), THEMA, Colombia, Tomo IV. Bogotá, 1990, p. 32-33. Para el caso de Cartagena existía en la década del 40 el Seminario de San Pedro donde los jóvenes de la ciudad podían recibir clases de carpintería, culinaria, panadería, música, entre otros. (Entrevista al músico Aníbal Montero Pájaro)

${ }^{29}$ AHC. El Porvenir. 13 de abril de 1927. 
${ }^{30}$ Silva, Renán. República intelectual liberal, intelectuales y cultura popular. La Carreta. Medellín, 2005.

31 Lemaitre, Alberto. Estampas de la Cartagena de ayer. Cartagena, 1994.

${ }^{32}$ Ibíd. págs. 37 y 107.

${ }^{33}$ García Canclini, Culturas híbridas, estrategias para entrar y salir de la modernidad. Grijalbo México. 2004. pág. 142. y mujeres. Solo bajó un poco a finales de la centuria, alcanzando los niveles de alfabetización de la Europa de finales del siglo XIX ${ }^{30}$.

Los esfuerzos del proyecto liberal de "desanalfabetizar" la población mediante las bibliotecas aldeanas, ambulantes y patronatos escolares (para la caridad), no resultaron muy satisfactorios, pese a la fuerte campaña de la radio como herramienta del Estado en un "programa cultural de masas" donde estas últimas eran vistas como "niños por educar y guiar". La radio nació en la década del 30 como consecuencia de la modernización de la ciudad. No obstante, el experimento liberal de utilizarla en su campaña de educación no tuvo mayor fuerza debido a que en ciudades como Cartagena no existían muchos aparatos para escuchar dichas "promociones", y de acuerdo a lo que dice Alberto Lemaitre, los cartageneros debían reunirse en el Camellón de los Mártires para escuchar la emisora "Ondas del Caribe" y en ella el programa "Tico Sico", todo un éxito de sintonía por su sentido del humor, y porque hablaba con el lenguaje de las clases populares de la época ${ }^{31}$.

Basados en estos hechos, se podría afirmar muy ligeramente que la prensa de Cartagena entre los años 1920-40, además de ser testigos de importantes cambios en la estructura de la ciudad -en teoría- solo creó un "espacio público" para un reducido grupo de lectores: la élite y un pequeño conjunto de personas con acceso a la instrucción educativa y con capacidad para leer y analizar la información contenida en los periódicos del período en cuestión. En este sentido, resulta evidente que el éxito de un medio de comunicación masivo consiste en contar con un amplio número de receptores directos, pero esto no implica, que para aquellos que no sabían leer y escribir, el ejercicio de la oralidad en las plazas y demás áreas comunes no les haya permitido tener contacto con las principales noticias que acontecían a nivel local y nacional, y que a su vez, eran registradas en los principales diarios cartageneros ${ }^{32}$.

La afirmación de Néstor García Canclini refuerza esta teoría cuando manifiesta que el público al que está dirigido este tipo de información no es homogéneo y de comportamiento constante. En él existen diferentes estratos económicos y educativos con hábitos culturales diferentes y donde la heterogeneidad de ofertas culturales permite la coexistencia de varias formas de recepción y comprensión ${ }^{33}$. En el caso de Cartagena, la oralidad se constituye como un medio en el que se difunden las ideas y noticias de carácter político o de interés colectivo. Por lo tanto, la socialización en la ciudad durante los años veinte al cuarenta resulta ser fundamental para la creación de un "espacio 
de opinión". Este "espacio público" en Cartagena se forjará en el seno de una sociedad con grandes desbalances culturales y/o económicos pero que en últimas, consolidará el papel del periodismo en la ciudad.

Como bien se advirtió al principio del trabajo, este es un primer acercamiento a la historia del periodismo en Cartagena, y al proceso que acompañó a la creación de un mercado masivo para los medios impresos en la ciudad, y la consecuente conformación de una opinión pública durante la primera mitad del siglo XX. Cabe anotar que aún queda muchísimo por explorar en un tema y un periodo histórico donde el peso de lo político o más bien de los partidos políticos tradicionales influían profundamente en todos los aspectos de la vida de los colombianos sin exceptuar la labor de los medios masivos de información existentes.

\section{BIBLIOGRAFIA}

AHC. Diario de la Costa, $\mathrm{n}^{\circ} 2508.6$ de octubre de 1.925.

AHC. El Porvenir, nº 7942. mayo de 1905.

AHC. El Porvenir. 13 de abril de 1927

AHC. El Porvenir. 16 de febrero de 1925.

AHC. El Porvenir. Jueves, 15 de enero de 1925.

AHC. Diario de la Cosa, no 2507, 5 de octubre de 1925.

El Universal, No 993. Junio 12 de 2005.

Álvarez, Jesús. Ascención Martínez. Historia de la prensa hispanoamericana colecciones MAPFRE. Edit. Madrid, 1992. 298 p.

Burke, Peter. La cultura popular en la Europa moderna. Alianza Editorial, Madrid, 464 pags

Chartier, Roger. El mundo como representación, Gedisa, Madrid.

García Canclini. Culturas híbridas, estrategias para entrar y salir de la modernidad. Grijalbo, México. 2004, 349 págs.

Hobsbawn, Eric. La Era del capital, 1848 - 1875. Buenos Aires, Crítica, 1998, 360 páginas.

Lemaitre, Alberto. Estampas de la Cartagena de ayer. Cartagena, 1994.

Lemaitre, Eduardo. Historia general de Cartagena, tomo IV. Banco de la República, Bogotá 1983.

PalObra No. 12. Agosto de 2010 - Julio de 2011 
Martínez, Germán y otros. "Filosofía de la Ilustración" en: La filosofía en Colombia, historia de las ideas, El Búho, edit., Bogotá, 1992.

(N.A.), THEMA, Colombia, Tomo IV. Bogotá, 1990.

Otero Muñoz, Gustavo. Historia del periodismo en Colombia, Selección Samper Ortega, Santa Fe de Bogotá, 1948.

Petit, Michele. Lectores: Del espacio íntimo al espacio público. Fondo de Cultura Económica. México, 2001, 176 pags

Santos, Adriana. "La prensa católica en el Estado Soberano del Magdalena: Guerra de palabras y pedagogía política, En: Taller de la Historia, № 2, Universidad de Cartagena, Facultad de Ciencias Humanas, 2002.

Silva, Renán. Prensa y revolucionaria a finales del s. XVIII. Banco de la República, Bogotá. 1988, 188 pags.

Silva, Renán. República intelectual Liberal, intelectuales y cultura popular. La Carreta. Medellín, 2005, 303 pags.

Zenón Davis, Nataly. "La imprenta y el pueblo", en: Sociedad y cultura en la Francia moderna, Crítica, Barcelona, págs. 186-224 


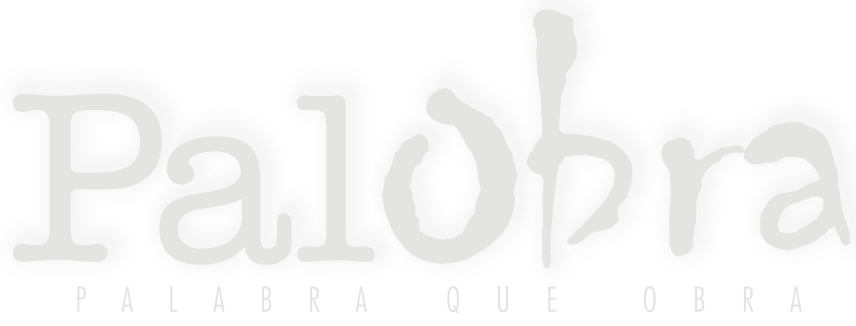

